\title{
Can ultrasound-guided infraclavicular block be an alternative option for forearm reduction in the emergency department? A prospective randomized study
}

\author{
Erdal Tekin ${ }^{1}$, Muhammed Enes Aydin ${ }^{2,3}$, Mehmet Cenk Turgut ${ }^{4}$, \\ Selahattin Karagoz ${ }^{1}$, Irem Ates ${ }^{2}$, Elif Oral Ahiskalioglu ${ }^{2,3}$ \\ 'Department of Emergency Medicine, Ataturk University School of Medicine, Erzurum, Turkey \\ ${ }^{2}$ Department of Anesthesiology and Reanimation, Ataturk University School of Medicine, Erzurum, Turkey \\ ${ }^{3}$ Clinical Research, Development and Design Application and Research Center, Ataturk University School of \\ Medicine, Erzurum, Turkey \\ ${ }^{4}$ Department of Orthopedic Surgery, Erzurum City Hospital, Erzurum, Turkey
}

Objective Ultrasound-guided infraclavicular nerve block (IB) has become a well-established method in several outpatient procedures; however, its use in emergency departments (EDs) remains limited. The aim of this study was to compare procedural sedation and anlagesia (PSA) and IB in the pain management for patients who underwent forearm fracture reduction in the ED.

Methods This prospective randomized study included 60 patients aged 18 to 65 years, who visited the ED with forearm fractures. They were randomly divided into two groups: Group PSA $(n=30)$ and Group IB $(n=30)$. The pain scores of patients were evaluated before and during the procedure with the visual analog scale. Complications and patient and operator satisfaction levels were recorded.

Results There was no difference between the two groups in terms of demographic characteristics. The median (interquartile range) pain scores observed during the procedures were significantly higher in Group PSA than in Group IB (4 [4-6] vs. 2 [0-2], respectively; $\mathrm{P}<0.001)$. Patient and operator satisfaction levels were significantly higher in Group IB $(P<0.001)$. Oxygen desaturation was statistically higher in Group PSA than in Group IB (40.00\% vs. $3.33 \%$, respectively; $\mathrm{P}=0.002)$.

Conclusion IB was an effective alternative for reducing pain and increasing patient satisfaction in ED patients undergoing forearm fracture reduction.

Keywords Fractures, closed; Trauma; Emergency service, hospital; Ultrasonography

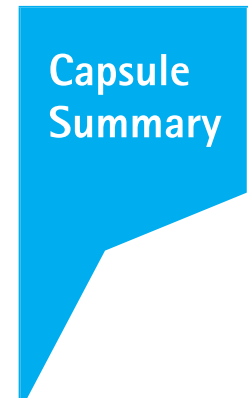

What is already known

Procedural sedation and analgesia (PSA) is commonly used analgesic method for forearm fracture reduction. The use of ultrasound-guided infraclavicular block is still limited in the emergency department.

What is new in the current study

Infraclavicular blocks can be used as an effective and safe analgesic method for adult forearm fractures reduction in the emergency department.
elSSN: 2383-4625

Received: 2 October 2020

Revised: 21 November 2020

Accepted: 23 November 2020

Correspondence to: Erdal Tekin Department of Emergency Medicine, Ataturk University School of Medicine, Erzurum 25240, Turkey E-mail:dret25@gmail.com ORCID

https://orcid.org/0000-0002-6158-0286

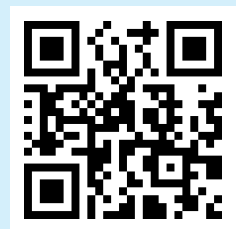

How to cite this article:

Tekin E, Aydin ME, Turgut MC, Karagoz S, Ates I, Ahiskalioglu EO. Can ultrasoundguided infraclavicular block be an alternative option for forearm reduction in the emergency department? A prospective randomized study. Clin Exp Emerg Med 2021;8(4):307-313. https://doi. org/10.15441/ceem.20.136

This is an Open Access article distributed under the terms of the Creative Commons Attribution Non-Commercial License (https:// creativecommons.org/licenses/by-nc/4.0/). 


\section{INTRODUCTION}

Trauma is a major cause of mortality and morbidity in young people. ${ }^{1}$ Forearm fractures are the most common upper extremity fractures in adult patients. Generally, upper extremity fractures are more common in males, whereas forearm fractures are more common in females. ${ }^{2,3}$ Patients with forearm fractures constitute $1.5 \%$ of cases in the emergency department (ED). ${ }^{4}$

Pain management is crucial in interventional procedures in EDs. Forearm fracture reduction is a painful procedure that often causes serious anxiety. Procedural sedation and anlagesia (PSA) is commonly employed in the ED to reduce both pain and anxiety. ${ }^{5-7}$ PSA helps to control reactions of patients to the environment and external stimuli and maintains their protective reflexes. However, it can sometimes lead to serious cardiopulmonary adverse effects depending on the comorbidities of patients and combinations of drugs. $^{8,9}$

Ultrasound (US)-guided peripheral nerve blocks are alternatives used in the pain management of forearm fracture reduction. ${ }^{10,11}$ These are commonly used in ambulatory and orthopedic surgeries in operating-room conditions and for postoperative pain management. ${ }^{11,12}$ In contrast, the frequency and indication of their use are increasing day by day in the EDs. ${ }^{13,14}$

The primary objective of this study was to compare the pain scores of PSA and US-guided infraclavicular nerve block (IB) in patients during forearm fracture reduction. The secondary objective was to compare the complications and patient and operator satisfaction between the two procedures.

\section{METHODS}

\section{Study design and adjustment}

This prospective randomized study was conducted in the ED of a tertiary university hospital. The study was approved by the ethics committee of Ataturk University, University Faculty of Medicine (No:8/3; date:27.12.2018). Informed consent for publication of the clinical images was obtained from the patient.

\section{Selection of participants}

For this study, we selected patients aged 18 to 65 years who were admitted to the ED with a forearm trauma between January 1 and May 31 2019; they were hemodynamically stable and were diagnosed with forearm fractures using standard radiography. We excluded patients who were under 18 years of age; were pregnant or lactating; were hemodynamically unstable; had forearm fractures requiring a surgical procedure; did not provide a written consent; had liver or renal insufficiency; were allergic to drugs used for sedation and anlagesia and peripheral nerve blockade; or had infections, open wounds, or coagulopathy in the area that would undergo blockage. Additionally, patients who could not be evaluated according to the visual analog scale (VAS) used in the study were excluded.

\section{Randomization}

Patients were randomly assigned to one of the two groups using a computer software program random 1:1 sequence (https://www. randomizer.org/\#randomize).

Group PSA $(n=30)$

Each patient was placed in the supine position, and peripheral vascular access was obtained. After examining their vital signs, PSA procedure was initiated. The following protocol was applied for PSA: fentanyl (Talinat $0.5 \mathrm{mg} / 10 \mathrm{~mL}$ vial; VEM Pharmaceutical Industry and Trade Corporation, Ankara, Turkey), $1 \mu \mathrm{g} / \mathrm{kg}$ intravenous (IV) infusion followed by propofol (propofol 2\% Fresenius 50 $\mathrm{mL}$ ampoule; Fresenius Kabi, Linz, Austria), 1 mg/kg IV infusion were administered. The decision to discharge patients was made according to the modified Aldrete score. ${ }^{15}$

\section{Group IB $(n=30)$}

Each patient was placed in the supine position, and peripheral vascular access was obtained. The head of the patient was turned to the opposite side of the area to be blocked. The arm that would be subjected to blocking was adducted and placed on the patient's chest in flexion. After the area of blockage was disinfected with polyvinylpyrrolidone-iodine, the US linear probe (Toshiba Aplio XG Diagnostic Ultrasound System; Toshiba Medicals, Tochigi, Japan) was longitudinally placed on the disinfected site for sterile administration of IB. On visualizing the axillary artery, vein, and brachial plexus cords, a 100-mm-long 22-G nerve stimulation needle (B. Braun, Geisingen, Germany) was guided towards the seven o'clock direction of the artery in the same plane as the US probe (Fig. 1). A mixture of $10 \mathrm{~mL} 2 \%$ lidocaine and $10 \mathrm{~mL}$ saline (with $1 / 200,000$ adrenaline) was prepared. First, $2 \mathrm{~mL}$ of saline was administered; when its spread was appropriate, the local anesthetic (LA) drug was injected with intermittent negative aspiration in fractionated doses. After 15 minutes of this procedure, the anesthesia level was examined using the cold-hot test, and the reduction and cast were applied.

\section{Study outcomes}

The pain severity levels of patients during admission and reduction were measured using a VAS: score $0=$ no pain and score $10=$ the most severe pain that can be felt. Patient and operator 

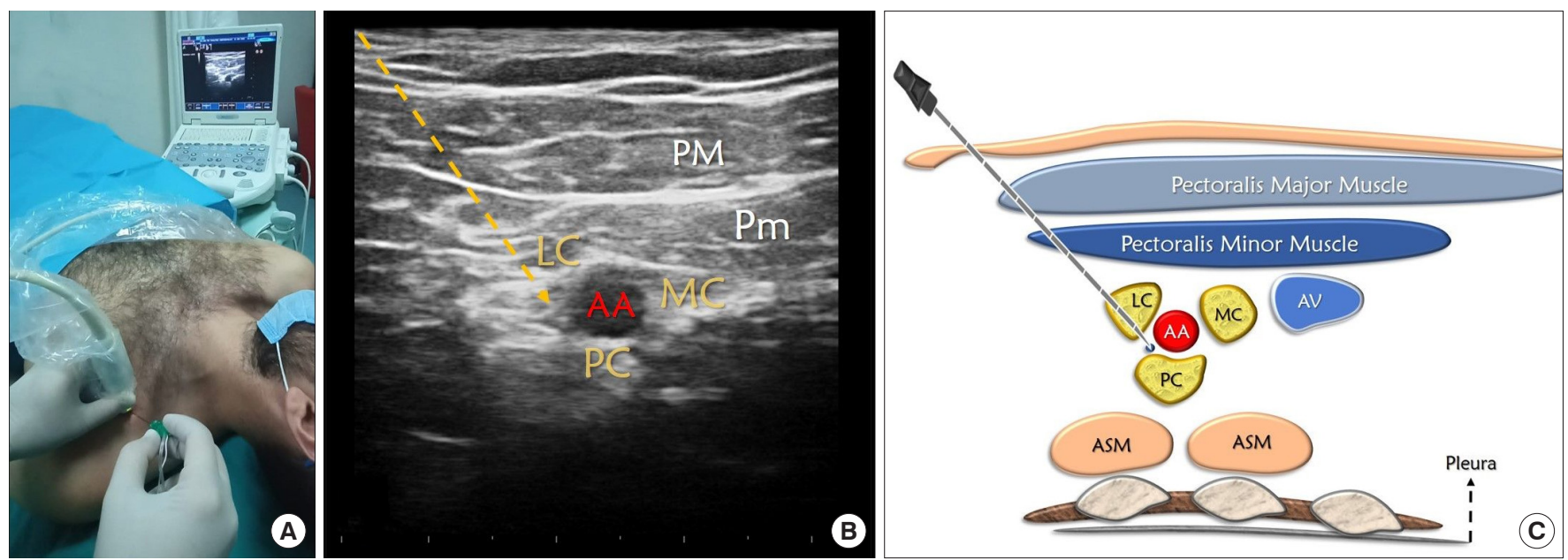

Fig. 1. Patient-ultrasound position in infraclavicular block, sonographic anatomy, and illustration view. (A) Ultrasound patient setup and needle orientation for infraclavicular block. (B) Sonographic anatomy of block. (C) Basic illustration of block. LC, lateral cord; AA, axillary artery; PC, posterior cord; MC, medial cord; PM, pectoralis major muscle; Pm, pectoralis minor muscle; ASM, anterior serratus muscle; AV, axillary vein.

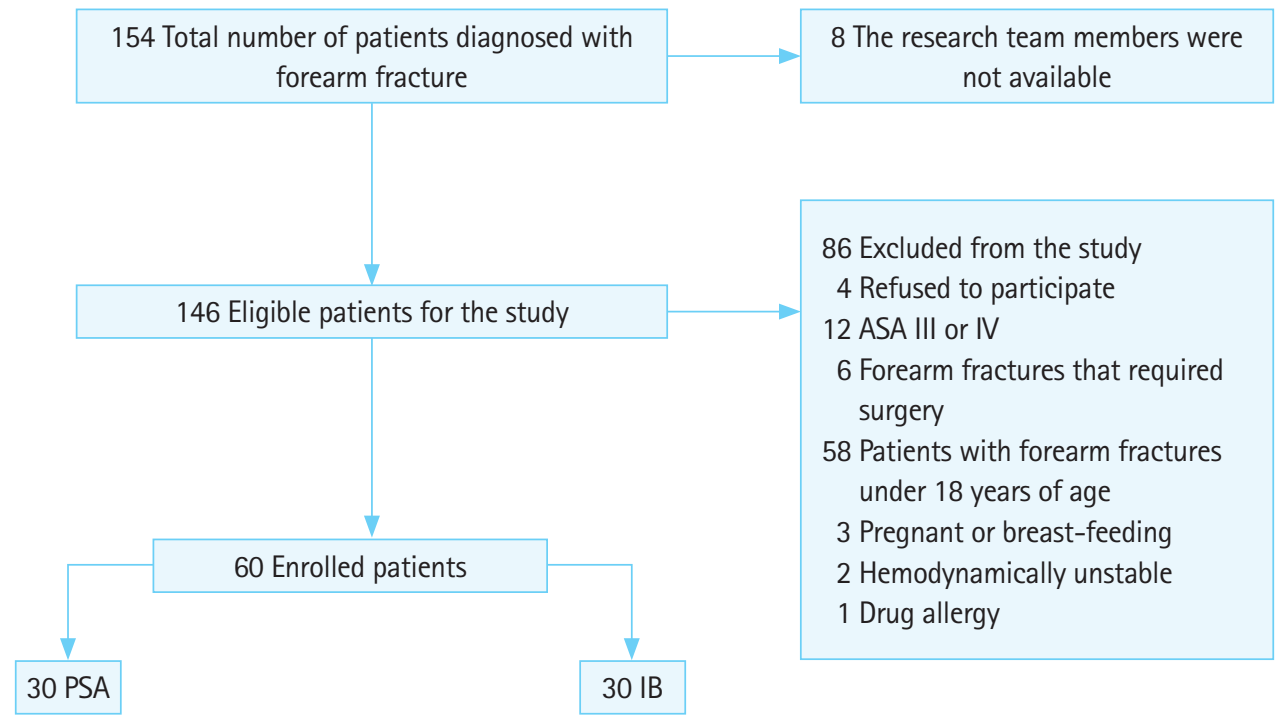

Fig. 2. Flowchart for inclusion of patients in the study. PSA, procedural sedation and anlagesia; IB, ultrasound-guided infraclavicular nerve block; ASA, American Society of Anesthesiologists.

satisfaction levels were evaluated on a scale of 1-4: $1=$ poor; $2=$ moderate; $3=$ good; and $4=$ excellent. All adverse effects during and after the procedure-local anesthetic systemic toxicity (LAST), hypotension, desaturation, respiratory depression, arrhythmia, and motor deficit-were recorded. Desaturation was considered as peripheral oxygen saturation $<90 \%$.

\section{Sample size}

A preliminary study was conducted to determine the ideal sample size for the study. This study indicated that our primary outcome of pain score using VAS during the procedure was $3.20 \pm 2.58$ in the IB group $(n=5)$ and $1.60 \pm 1.31$ in the PSA group $(n=5)$. A total sample size of 27 was determined for each group using $\mathrm{G}^{*}$ Power ver. 3.1.9.2 ${ }^{16}$ with an alpha probability of 0.05 , effect size 0.78 , and a power of 0.80 . Assuming possible dropouts, we included 30 patients in each group.

\section{Statistical analysis}

Statistical analysis was performed using IBM SPSS Statistics ver. 21.0 (IBM Corp., Armonk, New York, NY, USA). The distribution of variables was evaluated for normality using the Kolmogorov-Smirnov test. Normally distributed data were analyzed using the Student t-test, whereas, non normally distributed data were analyzed using the Mann-Whitney U-test. Categorical variables were 
Table 1. Demographic data of study

\begin{tabular}{lccc} 
Characteristics & $\begin{array}{c}\text { Group PSA } \\
(\mathrm{n}=30)\end{array}$ & $\begin{array}{c}\text { Group IB } \\
(\mathrm{n}=30)\end{array}$ & P-value \\
\hline Age $(\mathrm{yr})$ & $38.67 \pm 13.88$ & $44.23 \pm 15.40$ & $0.118^{\mathrm{a})}$ \\
Sex, male & 16 & 11 & $0.194^{\mathrm{b})}$ \\
Weight $(\mathrm{kg})$ & $75.13 \pm 10.49$ & $70.17 \pm 12.49$ & $0.184^{\mathrm{a})}$ \\
Height $(\mathrm{cm})$ & $169.67 \pm 9.60$ & $166.97 \pm 7.42$ & $0.173^{\mathrm{a})}$ \\
ASA, I:Il & $26: 4$ & $25: 5$ & $0.500^{\mathrm{c})}$ \\
Fracture localization, $\mathrm{R}: \mathrm{R}+\mathrm{U}$ & $24: 6$ & $26: 4$ & $\left.0.488^{\mathrm{c}}\right)$ \\
Fasting time (hr) & $3.97 \pm 2.24$ & $4.07 \pm 1.23$ & $0.375^{\mathrm{a}}$ \\
\hline
\end{tabular}

Values are presented as mean \pm standart deviation or number.

PSA, procedural sedation and anlagesia; IB, ultrasound-guided infraclavicular nerve block; ASA, American Society of Anesthesiologists; $R$, radius; $R+U$, radius and ulna.

${ }^{\text {a) } M a n n-W h i t n e y ~ U-t e s t . ~}{ }^{b}$ Chi-square test. ${ }^{\text {c) }}$ Fisher exact test.

Table 2. Pain scores, patient and operator satisfaction

\begin{tabular}{llccc}
\hline Variable & & $\begin{array}{c}\text { Group PSA } \\
(\mathrm{n}=30)\end{array}$ & $\begin{array}{c}\text { Group IB } \\
(\mathrm{n}=30)\end{array}$ & P-value \\
\hline VAS & Pre-procedure & $8(8-10)$ & $10(8-10)$ & $0.161^{\text {a) }}$ \\
\multirow{2}{*}{$\begin{array}{llc}\text { Patient satis- } \\
\text { faction }\end{array}$} & During reduction & $4(4-6)$ & $2(0-2)$ & $<0.001^{\text {a) }}$ \\
& Poor & 2 & 0 & $<0.001^{\text {b) }}$ \\
& Moderate & 13 & 1 & \\
\multirow{5}{*}{ Operator satis- } & Good & 13 & 3 & \\
faction & Poor & 2 & 26 & \\
& Moderate & 1 & 0 & $<0.001^{\text {b) }}$ \\
& Good & 14 & 1 & \\
& Excellent & 13 & 3 & \\
\hline
\end{tabular}

Values are presented as median (interquartile range) or number.

PSA, procedural sedation and anlagesia; IB, ultrasound-guided infraclavicular nerve block; VAS, visual analog scale.

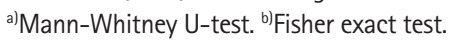

analyzed using the chi-square or Fisher exact test. $\mathrm{P}<0.05$ was considered statistically significant.

\section{RESULTS}

We screened 154 patients for the study. Eight patients were excluded due to the lack of appropriate anesthesiologists and orthopedic specialists in the study team, whereas 86 patients did not meet the inclusion criteria. We analyzed data for two groups of 30 patients each (Fig. 2). Table 1 summarizes the demographic data of patients. There was no statistically significant difference between the groups in terms of age, sex, weight, height, the American Society of Anesthesiologists (ASA) scores, fracture location, and fasting time $(P>0.05)$.

Table 2 displays the pain scores of patients and patient and operator satisfaction levels. There was no statistically significant difference between the groups in terms of pre-procedural pain
Table 3. Complications of study

\begin{tabular}{lccc}
\hline Complication & $\begin{array}{c}\text { Group PSA } \\
(\mathrm{n}=30)\end{array}$ & $\begin{array}{c}\text { Group IB } \\
(\mathrm{n}=30)\end{array}$ & P-value \\
\hline Desaturation & 12 & 1 & $0.002^{\mathrm{a})}$ \\
Nausea & 5 & 2 & $0.424^{\mathrm{b}}$ \\
Vomiting & 3 & 0 & $0.237^{\mathrm{b}}$ \\
Local anesthetics toxicity & 0 & 1 & $1.000^{\mathrm{b}}$ \\
Hypotension & 4 & 1 & $0.353^{\mathrm{b}}$ \\
Arrhythmia & 0 & 0 & $1.000^{\mathrm{b}}$ \\
\hline
\end{tabular}

Values are presented as number.

PSA, procedural sedation and anlagesia; IB, ultrasound-guided infraclavicular nerve block.

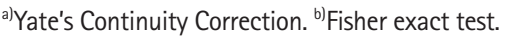

scores $(P=0.161)$, whereas during the procedure, Group PSA had significantly higher median (interquartile range) scores than Group IB (4 [4-6] vs. 2 [0-2], respectively; $P<0.001)$.

Table 3 summarizes the adverse effects observed in the study. In terms of adverse effects associated with the two procedures, we observed oxygen desaturation in 13 patients which was signifcantly higher in Group PSA than in Group IB (12/30 vs. 1/30, respectively; $P=0.002$ ). In addition, nausea, vomiting, and hypotension were more frequent in Group PSA, whereas one patient in Group IB experienced LA toxicity-related prodromal symptoms, manifesting in the form of a metallic taste in mouth. However, there was no difference between the groups in terms of these adverse effects $(P>0.05)$. No aspiration was observed in any of the three patients with vomiting in Group PSA.

\section{DISCUSSION}

Our study revealed that IB was more effective than PSA in terms of pain scores and patient and operator satisfaction during forearm fracture reduction in the ED. IB caused oxygen desaturation in significantly fewer patients than PSA.

Although anesthesiologists have extensively used it, other specialists have recently started employing PSA. ${ }^{8}$ Emergency physicians frequently use PSA in the diagnosis and treatment of patients, making it an indispensable part of EDs. Patients undergoing PSA should be monitored, and cardiopulmonary support equipment should be made available prior to the procedure. Although for patient safety it is necessary to provide similar conditions for those who undergo IB, the incidence of systemic adverse effects is higher with agents used for PSA than those used for IB. Such incidences increase with shorter fasting time and existing comorbidities.

During PSA, protective reflexes that provide airway patency are often unaffected, but patients may not be maintained at the re- 
quired levels of deep sedation. Patients are faced with the risk of respiratory depression and aspiration. To minimize the risk of aspiration, the ASA has defined fasting times for patients. ${ }^{17}$ In contrast, the American College of Emergency Physicians stated that the risk of vomiting, loss of airway protective reflexes, and aspiration during PSA is extremely rare and emphasized that administration of PSA in the ED should not be delayed due to limited fasting time. ${ }^{18-20}$ In this study, we aimed to attain conscious sedation levels in the PSA group to better evaluate the pain score without suppressing airway reflexes.

The forearm fracture reduction should be performed immediately to minimize damage to vascular and neural structures that may develop in such fractures. ${ }^{21}$ Thus, the fasting status of patients undergoing forearm fracture reduction in the ED may not be optimized for PSA; instead, regional anesthesia techniques are often preferred to avoid PSA or general anesthesia in patients. This study demonstrates the advantage of the IB method over PSA from the standpoint of patients and physicians in emergency interventions. In this study, although there were no significant differences in fasting time between the two groups, aspiration was not observed in any of these patients since the depth of sedation achieved was moderate; however, vomiting occurred in three patients in the PSA group.

Propofol and fentanyl are commonly preferred agents for achieving PSA with rapid onset and short action time. Both agents are safe and effective at appropriate clinical dosages. ${ }^{22-24}$ However, considering the urgency of procedures in the ED and comorbidities of patients, they may cause serious cardiac and respiratory adverse effects. $^{8,23,25}$ Thus, we opted to use $1 \%$ lidocaine in the IB procedure due to rapid onset of action and appropriate action time (30-60 minutes) for rapid discharge from the ED. Additionally, its use enabled us to immediately recognize adverse effects related to casting after the reduction; we did not have to rely on a combination with a long-acting $L A$ because the pain decreased after the reduction.

PSA is an essential application in interventional procedures and pain management in EDs, and reduces workload of health practitioners. ${ }^{9}$ However, with PSA procedures, rapid removal of a patient from the room is not possible due to the need for ongoing monitoring and oxygen administration. Vital signs of patients undergoing PSA should be monitored closely in the ED until they are awake and alert. This situation causes delays in planned operations and space occupation. Since patients undergoing IB are not sedated, early mobilization is feasible after the forearm fracture reduction.

The use of US in regional anesthesia has now enabled safer implementation of nerve blocks. This trend has also been reflected in EDs, with emergency physicians safely performing nerve blocks. The IB method may cause serious complications, such as pneumothorax, due to its proximity to pulmonary structures. However, the possibility of such an event is drastically reduced due to adequate analysis of the sonographic anatomy with US guidance. The supraclavicular block is preferred for the upper extremity distal to surgical procedures of the shoulder; in contrast, IB is preferred for operations of the distal arm, elbow, wrist, and hand. IB is easy to learn and apply.

Due to its possible adverse effects such as nerve damage and LAST, IB has not been widely accepted in ED practice. However, the likelihood of LAST is rare, although it varies according to the dose of LA and its mode of injection. 12,26,27 Studies conducted in adults have demonstrated the feasibility of low doses of LA for successful IB. ${ }^{28,29}$ Additionally, nerve damage is extremely rare in IB. ${ }^{29}$ In our study, we used the appropriate clinical doses of LA to avoid LAST. In addition, we aimed to prevent any adverse effects of IB by avoiding intravascular and intraneural injections.

In the $E D$, pain management is important in patients with early stages of trauma, resulting in the frequent use of opioids such as tramadol, meperidine $\mathrm{HCl}$ and fentanyl. In patients with bone fractures, the need for analgesia is required after imaging. Peripheral nerve blocks should be used to ensure their comfort and to reduce the use of opioid drugs. ${ }^{30,31}$ Our study demonstrates that excellent results can be obtained by using low doses of LA in upper extremity fractures.

Our study has some limitations. First, in the study, although we achieved an effective reduction in pain during the forearm fracture reduction, we did not investigate pain scores related to the infraclavicular block procedure. Second, oxygen desaturation occurred more frequently in patients who underwent PSA than those who underwent IB, due to the use of propofol and fentanyl; desaturation events could be reduced if agents such as ketamine were used. Third, this was not a blinded study due to its nature; the attendant who evaluated the results could identify the patients' interventions according to their level of consciousness. Fourth, we did not evaluate the long-term outcomes of patients. Fifth, the study was conducted without the assessment of the cost-effectiveness of IB; this method could be expensive due to the high cost of the needle.

In conclusion, IB may be preferred over PSA to increase both patient and operator satisfaction in EDs. Although both methods reduce pain, IB is more effective with fewer adverse events.

\section{CONFLICT OF INTEREST}

No potential conflict of interest relevant to this article was reported. 


\section{REFERENCES}

1. Mercier E, Cameron PA, Smith K, Beck B. Prehospital trauma death review in the State of Victoria, Australia: a study protocol. BMJ Open 2018;8:e022070.

2. Wu JC, Strickland CD, Chambers JS. Wrist fractures and osteoporosis. Orthop Clin North Am 2019;50:211-21.

3. Macintyre NJ, Dewan N. Epidemiology of distal radius fractures and factors predicting risk and prognosis. J Hand Ther 2016;29:136-45.

4. Chung KC, Spilson SV. The frequency and epidemiology of hand and forearm fractures in the United States. J Hand Surg Am 2001;26:908-15.

5. Miguez Navarro C, Oikonomopoulou N, Rivas Garcla A, et al. Efficacy, safety and satisfaction of sedation-analgesia in Spanish emergency departments. An Pediatr (Barc) 2019;90:32-41.

6. Celis-Rodriguez $E$, Birchenall $C$, de la Cal MA, et al. Clinical practice guidelines for evidence-based management of sedoanalgesia in critically ill adult patients. Med Intensiva 2013; 37:519-74.

7. Mora Capin A, Miguez Navarro C, Lopez Lopez R, Maranon Pardillo R. Usefulness of capnography for monitoring sedoanalgesia: influence of oxygen on the parameters monitored. An Pediatr (Barc) 2014;80:41-6.

8. Ramalho CE, Bretas PM, Schvartsman C, Reis AG. Sedation and analgesia for procedures in the pediatric emergency room. J Pediatr (Rio J) 2017;93 Suppl 1:2-18.

9. Maghraby N, Pearson E, Xue X, Colacone A, Afilalo M. What is the impact of the implementation of an evidence based procedural sedation protocol in the emergency department? J Clin Trials 2016;6:282.

10. Chin KJ, Alakkad H, Adhikary SD, Singh M. Infraclavicular brachial plexus block for regional anaesthesia of the lower arm. Cochrane Database Syst Rev 2013;(8):CD005487.

11. Salinas FV, Joseph RS. Peripheral nerve blocks for ambulatory surgery. Anesthesiol Clin 2014;32:341-55.

12. Koscielniak-Nielsen ZJ. Ultrasound-guided peripheral nerve blocks: what are the benefits? Acta Anaesthesiol Scand 2008; 52:727-37.

13. Frenkel 0, Liebmann 0, Fischer JW. Ultrasound-guided forearm nerve blocks in kids: a novel method for pain control in the treatment of hand-injured pediatric patients in the emergency department. Pediatr Emerg Care 2015;31:255-9.

14. Ritcey B, Pageau P, Woo MY, Perry JJ. Regional nerve blocks for hip and femoral neck fractures in the emergency department: a systematic review. CJEM 2016;18:37-47.

15. Faul $F$, Erdfelder $E$, Buchner A, Lang AG. Statistical power analyses using $\mathrm{G}^{*}$ Power 3.1 : tests for correlation and regression analyses. Behav Res Methods 2009;41:1149-60.

16. Hinkelbein J, Lamperti M, Akeson J, et al. European Society of Anaesthesiology and European Board of Anaesthesiology guidelines for procedural sedation and analgesia in adults. Eur J Anaesthesiol 2018;35:6-24.

17. Practice guidelines for preoperative fasting and the use of pharmacologic agents to reduce the risk of pulmonary aspiration: application to healthy patients undergoing elective procedures. An updated report by the American Society of Anesthesiologists Task Force on preoperative fasting and the use of pharmacologic agents to reduce the risk of pulmonary aspiration. Anesthesiology 2017;126:376-93.

18. Godwin SA, Burton JH, Gerardo CJ, et al. Clinical policy: procedural sedation and analgesia in the emergency department. Ann Emerg Med 2014;63:247-58.

19. Roback MG, Green SM, Andolfatto G, Leroy PL, Mason KP. Tracking and Reporting Outcomes Of Procedural Sedation (TROOPS): standardized quality improvement and research tools from the International Committee for the Advancement of Procedural Sedation. Br J Anaesth 2018;120:164-72.

20. Green SM. Fasting is a consideration--not a necessity--for emergency department procedural sedation and analgesia. Ann Emerg Med 2003;42:647-50.

21. Bowman EN, Mehlman CT, Lindsell CJ, Tamai J. Nonoperative treatment of both-bone forearm shaft fractures in children: predictors of early radiographic failure. J Pediatr Orthop 2011; 31:23-32.

22. Chiaretti A, Benini F, Pierri F, et al. Safety and efficacy of propofol administered by paediatricians during procedural sedation in children. Acta Paediatr 2014;103:182-7.

23. Milius EM, Papademetrious TR, Heitlinger LA. Retrospective review of propofol dosing for procedural sedation in pediatric patients. J Pediatr Pharmacol Ther 2012;17:246-51.

24. Godambe SA, Elliot V, Matheny D, Pershad J. Comparison of propofol/fentanyl versus ketamine/midazolam for brief orthopedic procedural sedation in a pediatric emergency department. Pediatrics 2003;112(1 Pt 1):116-23.

25. Fleischman RJ, Frazer DG, Daya M, Jui J, Newgard CD. Effectiveness and safety of fentanyl compared with morphine for out-of-hospital analgesia. Prehosp Emerg Care 2010;14:16775.

26. Guay J. Adverse events associated with intravenous regional anesthesia (Bier block): a systematic review of complications. J Clin Anesth 2009;21:585-94.

27. Gurich RW Jr, Langan JW, Teasdall RJ, Tanner SL, Sanders JL. Tourniquet deflation prior to 20 minutes in upper extremity 
intravenous regional anesthesia. Hand (N Y) 2018;13:223-7.

28. Sandhu NS, Bahniwal CS, Capan LM. Feasibility of an infraclavicular block with a reduced volume of lidocaine with sonographic guidance. J Ultrasound Med 2006;25:51-6.

29. Sandhu NS, Maharlouei B, Patel B, Erkulwater E, Medabalmi P. Simultaneous bilateral infraclavicular brachial plexus blocks with low-dose lidocaine using ultrasound guidance. Anesthesiology 2006;104:199-201.
30. Stone MB, Wang R, Price DD. Ultrasound-guided supraclavicular brachial plexus nerve block vs procedural sedation for the treatment of upper extremity emergencies. Am J Emerg Med 2008;26:706-10.

31. Heflin T, Ahern T, Herring A. Ultrasound-guided infraclavicular brachial plexus block for emergency management of a posterior elbow dislocation. Am J Emerg Med 2015;33:1324. 